\title{
An Efficient Denoising Architecture for Impulse Noise Removal in Colour Image Using Combined Filter
}

\author{
S. Arul Jothi ${ }^{*}$, N. Santhiya Kumari ${ }^{2}$, M. Ram Kumar Raja ${ }^{3}$ \\ ${ }^{1}$ ECE Department, Sri Ramakrishna Engineering College, Coimbatore, Tamilnadu. \\ ${ }^{2}$ ECE Department, Knowledge Institute of Technology, Salem,Tamilnadu. \\ ${ }^{3}$ ECE Department, Coimbatore Institute of Engineering and Technology, Coimbatore, Tamilnadu. \\ * Corresponding author. Email: aruljothiphd@gmail.com \\ Manuscript submitted December 18, 2015; accepted March 12, 2016. \\ doi: 10.17706/jsw.11.5.455-463
}

\begin{abstract}
Two main tasks of image processing are noise filtering and image enhancement.During image acquisition and transmission the images are corrupted by noise.these noise will affect the performance of image processing mehods, herefore to improve the performance efficient denoising technique is necessary for image processing methods.In this paper the performance of combined filter in impulse denoising has been compared with the marginal median filter.the statistical values such as PSNR,MSE,MAE are used to analyze the performance of the filterswith he aid of MATlab software. This combined filter (Resampling and MAMMF) removes the aliasing, blurring and impulse noise problem and produce the highest quality images with low cost and complexity.
\end{abstract}

Key words: Combined filer, impulse noise, MAE, MMF, MSE, PSNR.

\section{Introduction}

Image processing is widely used in many applications and research area such as visualization, image sharpening and restoration, image retrieval, image recognition and measurement of patterns [1]. In general, images are corrupted by some of the scaling factors and impulse noise during the image attainment, transformation [2]. This noise in the images may affect the image processing results and performance measures. So, that before processing the original image, scaling and noise removal techniques are applied to the image for obtaining the best performance result. Image scaling is process of resizing the images and it is non-trivial process which is tradeoff between efficiency, sharpness, smoothness. Image scaling algorithm is distinct into polynomial, and non-polynomial method. The polynomial based method is simple and also low complexity, even though it gives the highest quality output image [3]. The output of the scaled image has some of the impulse noise, so it has to be removed by impulse noise removal techniques. There are several noise reduction filters such as conventional smoothening filters; median filters are used to remove the impulse noise in the digital image [4]. But this smoothening and median filter is not enough when the images have high levels of noise. So in this proposed system, Modified Resampling filters use for image scaling and impulse related noise is removed from image using Modified Adaptive Marginal Median Filter. Resizing or scaling is the process of changing the size of the image which leads to several effects in the original image [5]. The following Fig.1 shows that the sample resize image. 

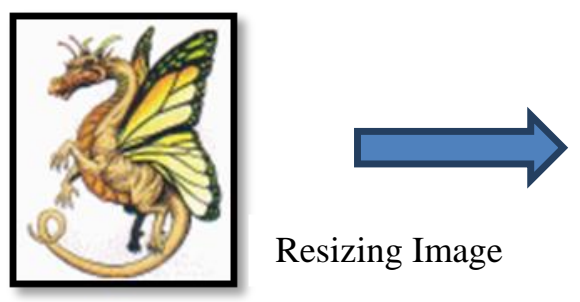

Resizing Image

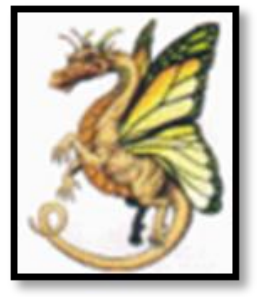

Fig. 1. Sample image.

In the above Fig. 1, the scaled (resized) image does not look like the original image; this will affects the further image processing analysis. For overcoming this problem, resampling filter will be used. In this filter first image has to be resized, and then the original image pixel value has to be placed in the correct place by Hausdorff based distance measure. After that the interpolate value is calculated by Lanczos filters which use the convolution kernel. This filter calculates the input image values rather than the output image, so it provides the best result when scaling the image. From the resultant image, impulse noise has been removed by modified adaptive marginal median filter. This MAMMF filter is easy to use and it produces the quality improved output image.The following section describes that related works of image scaling and impulse noise removal method, proposed system, result and discussion.

\section{Related Work}

Image Processing is one of the main technique which is used to process the images for analyzing particular sharpening and restoration information. But one of the major challenges is, the original image may be corrupted by scaling factors and impulse noise. Pei-Yin Chen et al., [6] focuses on VLSI implementation of high quality image with low cost,proposes the Simple Edge Preserved Denoising (SEPD) technique to reduce the impulse noise in the image. Based on the result, Quantitative Analysis and Visual Quality are used to evaluate the performance. Pei-Yin Chen [7] presents the quality image with low cost image scaling process. The author Describes the Edge Oriented - Area based Pixel Scaling to preserve the image features for obtaining the quality image. In the system, performance is evaluated based on both subjective and objective rather than the low complexity scaling methods. Chih-Yuan Lien et al., [8] discussed about efficiently removing the impulse noise from the image by decision tree based impulse noise detector and edge preserving filter. In this system noisy image is detected by using decision tree and then the noise oriented edge is filter by edge preserving filter. This combined technique is used to achieve the quality image and the performance is evaluated based on quantitative analysis, visual quality. Shih-Lun Chen et al., [9] focuses on VLSI implementation of high quality image scaling processor. In this proposed system bilinear interpolation for smoothing the image after resizing. This filter combined to work with the pre filter like sharpening filter and clamp filter for removing the aliasing and blurring problem. And also for reducing the memory T-model and inverse T-model convolution kernel is used to improve the quality of the image with low hardware cost. M. Emin Yuksel et al., [10] focuses on reducing the blur and impulse noise removal by Neuro fuzzy based decision maker. In the proposes system Neuro - fuzzy system has detected the noise in the image with help of the artificial training image. Based on the training result decision making detector will identify the impulse noise in the original image and remove those noises by using filters.

\section{Proposed Works}

Image processing has played an important role in daily life, such as computer tomography, satellite television, magnetic resonance [11]. But the images are corrupted by different type of noise and scaling factors, during the image capturing and transmission time. So in the proposed system resampling and modified adaptive marginal median filter is used to overcome the problem in a digital image. Following 
block diagram shows that the proposed scaling and impulse noise removal algorithm.

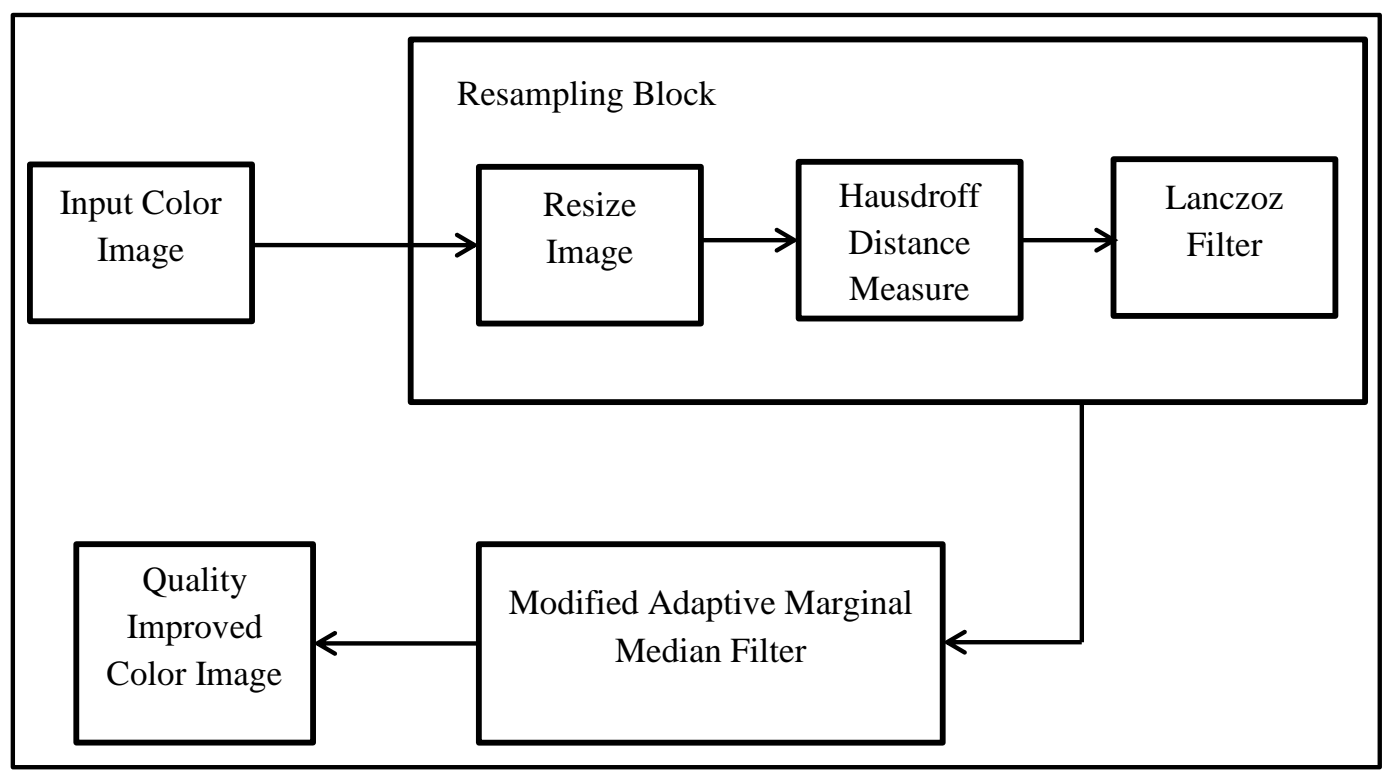

Fig. 2. Proposed block diagram.

In the above proposed diagram, first input color image has been resized and then the related pixel value is placed by hausdroff distance measure. The resulting image is smoothen by Lanczos filter. The Lanczos filter uses the convolution kernel which calculates the interpolated values of the input image rather than the output image. Finally, this process gives the scaled image, but it has some of the impulse noise which is reducing the quality of the image. For removing impulse noise in the image, Modified Adaptive Marginal Median Filter is used. The MAMMF filter gives the enhanced quality color image. The following section explains the details of proposed scaling and impulse noise removal algorithm.

\subsection{Proposed Image Scaling by Resampling Filter}

Image scaling is the process of converting the image dimension from one resolution to another resolution without losing the visual quality of the image [12]. Image scaling algorithm is divided into adaptive and non-adaptive method. The proposed system uses the non-adaptive method which provides lower cost and high quality based image. In non- adaptive method, image can be resized by either high resolution to low resolution or low to high resolution. During the image scaling process input image (original image) considered as the base image and the pixel value of the original image is compared with the pixel value of the resized image in order to determine the correctness of the new pixel value. This can be achieved by using distance measure. So, in the proposed system Hausdroff distance is used to calculate the distance between the pixels. Equation (1) is used to calculate the distance between the neighbor pixel values.

$$
d_{H}(X, Y)=\max \left\{\sup _{x \in X} \inf _{y \in Y} d(x, y), \sup _{y \in Y} \inf _{x \in X} d(x, y)\right.
$$

Sup- supremum, inf- infimum.

where,

- New Image

$d(x, y)$ - Original Image

Based on the above equation, new images generated, and then the interpolated values are determined by using a Lancozs interpolation filter with kernel values in order to avoid aliasing effects. This filter is sinc function windowed by central lobe, which is used to calculate the scaled kernel at the desired point. The 
Lancozes kernel function [13] is calculated using following equation (2).

$$
L(X)=\left\{\begin{array}{ccc}
\operatorname{sinc}(x) \operatorname{sinc}\left(\frac{x}{a}\right) & \text { if }-a<x<a \\
0 & \text { otherwise }
\end{array}\right.
$$

Here $a$ is the positive number, which is used to identify the size of the kernel value. The above process gives the enhanced scaled image.

\subsection{Proposed Impulse Noise Removal - MAMMF Algorithm}

The enhanced scaled image has some of the noise. The noise may be impulse and Gaussian noise. This noise will affect the image quality. [14]. The noise may be removed by using linear and non-linear method. In this proposed system impulse noise is removed by non- linear method. It proposes the Modified Adaptive Marginal Median non-linear filter uses for impulse noise removal. The Marginal Median Filter is merged to work with the Vector Median Filter (VMF) [15]. The vector median filter removing noise from the image by selecting the group of pixel in each filtering window. So, in the proposed system first select the group of pixel using fixed set of coordinates and then check the difference between the central pixel with a neighboring pixel is used to identify the central pixel as noise. If the central pixel value is corrupted by noise, then sort all the pixel values. And then find the marginal median value which is used to replace the corrupted pixel value. In adaptive marginal median filter, median value is placed between the minimum and maximum value of the pixel list. Then the output of the AMMF will be checked against the impulse residual noise by the distance between the nearest pixel using the minkowsi distance measure. Then the corrupted pixel is replaced by the modified adaptive marginal median filter value. This impulse noise removal process changes only the corrupted pixel and then remaining pixel will be unchanged.

\section{Result and Discussion}

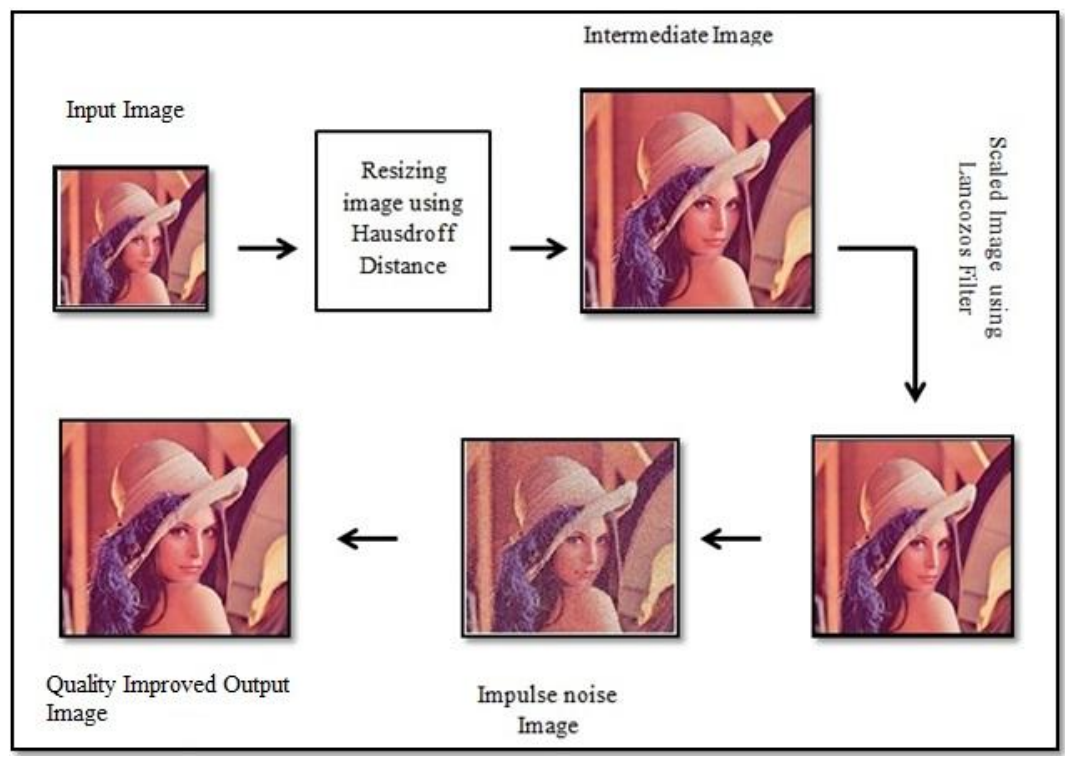

Fig. 3. Proposed algorithm based result.

The Performance of the proposed algorithm and quality of the noise free scaled image is measured by using PSNR and mean square error extractor. Following Fig. 3 shows that the sample result when applying the proposed algorithm to the color input image. 
From the enhance output image, performance of the proposed algorithm is calculated by using PSNR, MSE, MAE. PSNR, MAE and MSE is calculated [16] by using following equation (3),(4) and (5).

$$
P N S R=10 \log _{10}\left(\frac{M A X_{I}^{2}}{M S E}\right)
$$

where $M A X I$ is the maximum possible pixel value of the image.

$$
M S E=\frac{1}{m n} \sum_{y=1}^{n} \sum_{x=1}^{m}\left[I(x, y)-I^{\prime}(x, y)\right]^{2}
$$

The size of the image is $n X m$. Then the Mean Absolute Error value is calculated by using the following equation.

$$
M A E=\frac{1}{m n} \sum_{y=1}^{n} \sum_{x=1}^{m}\left[I(x, y)-I^{\prime}(x, y)\right]
$$

Following Table 1 shows that the different PSNR, MAE, MSE values of the proposed algorithm for various images which are shown in following Fig. 4.

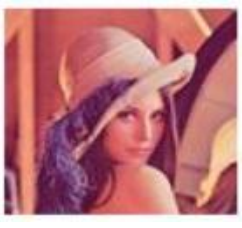

lena

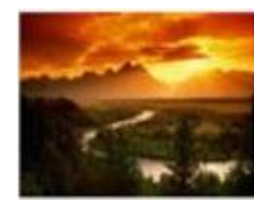

Sun rise

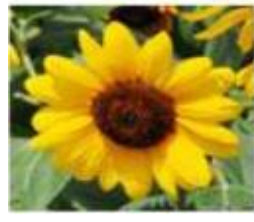

flower

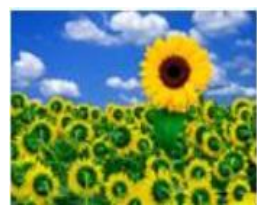

Flower1

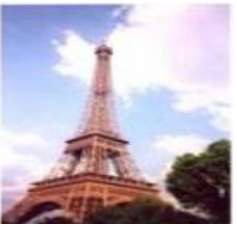

tower

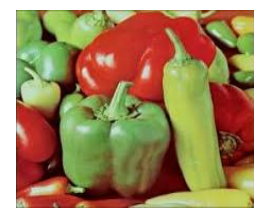

pepper

Fig. 4. Various images used for proposed algorithm.

Table 1. Proposed Algorithm Quality Measures

\begin{tabular}{|c|c|c|c|c|c|c|}
\hline Images & \multicolumn{5}{|c|}{ MMF } & \multicolumn{3}{c|}{ Resampling and MAMMF } \\
\hline & PSNR (db) & $\begin{array}{c}\text { MSE (pixel } \\
\text { square) }\end{array}$ & $\begin{array}{c}\text { MAE (pixel } \\
\text { square }\end{array}$ & PSNR (db) & $\begin{array}{c}\text { MSE (pixel } \\
\text { square) }\end{array}$ & $\begin{array}{c}\text { MAE(pixel } \\
\text { square) }\end{array}$ \\
\hline Lena & 38.2416 & 227.756 & 13.365 & 44.5834 & 225 & 10.334 \\
\hline Flower & 37.036 & 265.36 & 15.36 & 43.369 & 236.24 & 13.265 \\
\hline Tower & 38.36 & 265.36 & 14.36 & 45.365 & 241.3 & 12.365 \\
\hline Sun Rise & 31.23 & 243.25 & 15.36 & 43.21 & 231.445 & 14.368 \\
\hline Flower1 & 36.26 & 256.3 & 13.26 & 41.032 & 246.12 & 11.023 \\
\hline Pepper & 37.569 & 264.369 & 14.06 & 46.31 & 256.26 & 12.01 \\
\hline
\end{tabular}


From the above figure 4the proposed system quality measure is estimated with the help of the PSNR,MSE and MAE values which is shown in following Table 1.

From the table the original image based PSNR, MAE and MSE values are calculated with the help of the proposed scaling and impulse noise removal algorithm which is used to identify the highest quality image with low cost. The Table 2 shows that the different noise level based quality measure values based on the several image.

Table 2. Different Noise Level based Quality Measure

\begin{tabular}{|c|c|c|c|c|c|c|c|}
\hline \multirow[t]{2}{*}{$\begin{array}{c}\text { Noise } \\
\text { Level (\%) }\end{array}$} & \multirow[t]{2}{*}{ Images } & \multicolumn{3}{|c|}{ Marginal Median Filter (MMF) } & \multicolumn{3}{|c|}{$\begin{array}{c}\text { Modified Adaptive Marginal Median } \\
\text { Filter (MAMMF) }\end{array}$} \\
\hline & & PSNR & MSE & MAE & PSNR & MSE & MAE \\
\hline \multirow{6}{*}{$10 \%$} & Lena & 35.241 & 225.756 & 18.365 & 42.583 & 223 & 15.334 \\
\hline & Flower & 37.036 & 247.36 & 17.36 & 44.369 & 233.24 & 13.265 \\
\hline & Tower & 38.36 & 267.36 & 15.36 & 45.365 & 245.3 & 12.365 \\
\hline & Sun Rise & 33.23 & 243.25 & 17.36 & 43.21 & 235.445 & 14.368 \\
\hline & Flower1 & 36.26 & 256.3 & 14.26 & 41.032 & 246.12 & 11.023 \\
\hline & Pepper & 37.569 & 264.369 & 14.06 & 46.31 & 256.26 & 12.01 \\
\hline \multirow{6}{*}{$20 \%$} & Lena & 34.09 & 235.42 & 17.901 & 36.43 & 220.2 & 16.34 \\
\hline & Flower & 35.02 & 237.9 & 16.022 & 37.09 & 229.3 & 14.03 \\
\hline & Tower & 36.32 & 239.12 & 15.21 & 38.92 & 232.98 & 13.21 \\
\hline & Sun Rise & 31.01 & 232.0 & 15.20 & 34.42 & 224.1 & 13.20 \\
\hline & Flower1 & 32.54 & 233.98 & 13.24 & 33.65 & 231.67 & 12.01 \\
\hline & Pepper & 34.87 & 253.13 & 13.98 & 36.96 & 242.2 & 11.02 \\
\hline \multirow{6}{*}{$30 \%$} & Lena & 33.01 & 278.92 & 15.09 & 38.09 & 245.09 & 13.2 \\
\hline & Flower & 33.29 & 284.3 & 13.34 & 40.01 & 254.3 & 11.32 \\
\hline & Tower & 32.09 & 259.3 & 16.98 & 37.90 & 243.09 & 14.23 \\
\hline & Sun Rise & 29.98 & 264.3 & 14.67 & 35.89 & 224.6 & 12.43 \\
\hline & Flower1 & 30.87 & 256.7 & 13.89 & 35.85 & 232.54 & 11.21 \\
\hline & Pepper & 32.23 & 294.3 & 12.79 & 38.97 & 263.65 & 11.91 \\
\hline \multirow{6}{*}{$40 \%$} & Lena & 29.1 & 298.3 & 14.21 & 32.01 & 262.31 & 13.02 \\
\hline & Flower & 31.09 & 301.2 & 13.32 & 34.21 & 273.32 & 12.34 \\
\hline & Tower & 30.42 & 287.5 & 14.25 & 35.041 & 263.2 & 12.31 \\
\hline & Sun Rise & 28.31 & 299.7 & 13.24 & 30.21 & 259.01 & 11.32 \\
\hline & Flower1 & 29.34 & 302.12 & 12.43 & 34.09 & 254.2 & 10.32 \\
\hline & Pepper & 29.01 & 312.4 & 12.53 & 35.98 & 280.34 & 10.01 \\
\hline \multirow{6}{*}{$50 \%$} & Lena & 25.72 & 301.2 & 13.02 & 32.09 & 283.2 & 11.54 \\
\hline & Flower & 28.91 & 312.3 & 12.32 & 30.12 & 290.32 & 11.002 \\
\hline & Tower & 26.32 & $315 . .32$ & 13.21 & 29.12 & 289.4 & 12.10 \\
\hline & Sun Rise & 26.34 & 342.1 & 12.43 & 30.12 & 284.3 & 11.02 \\
\hline & Flower1 & 27.3 & 314.32 & 11.45 & 32.76 & 273.4 & 10.2 \\
\hline & Pepper & 26.3 & 315.2 & 10.23 & 29.34 & 312 & 9.34 \\
\hline
\end{tabular}

So, that the image scaling process is combined with the impulse noise removal method the system will produce the high quality enhanced output image with low complexity. The following Table 3 shows that the combine filter values for analyzing the performance. 


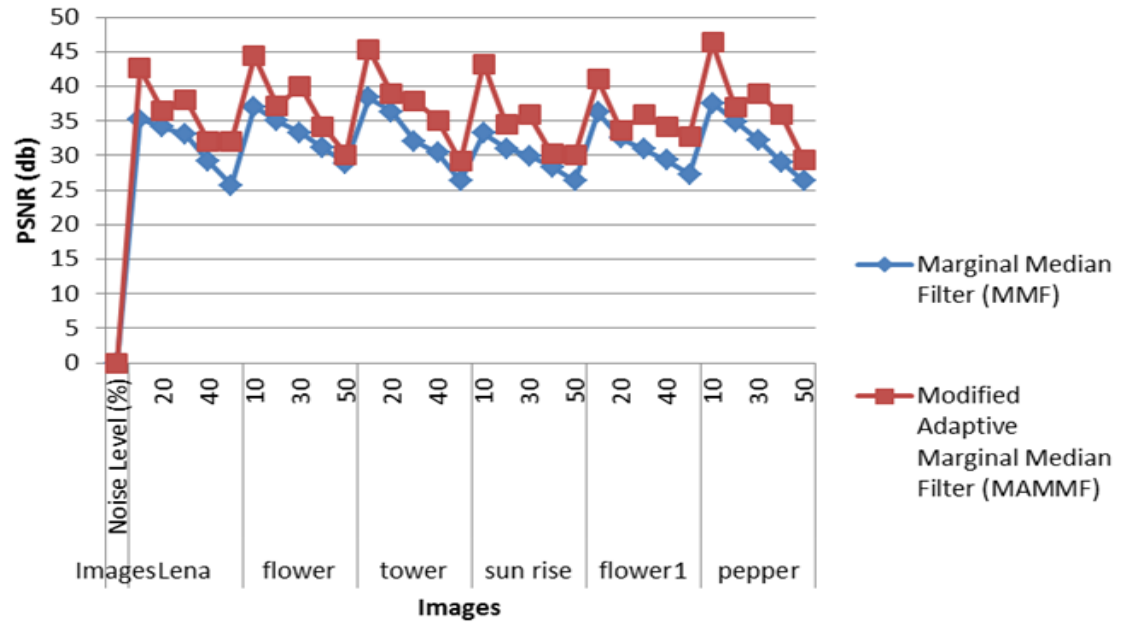

Fig. 7. PSNR for different level of noise.

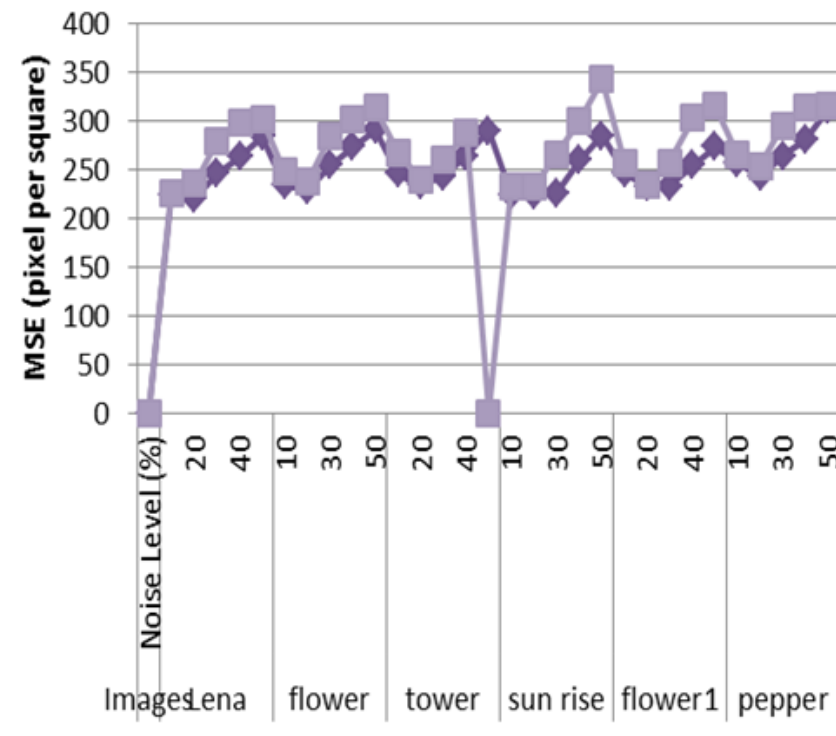

Images

Fig. 8. MSE for diffeent level noise.

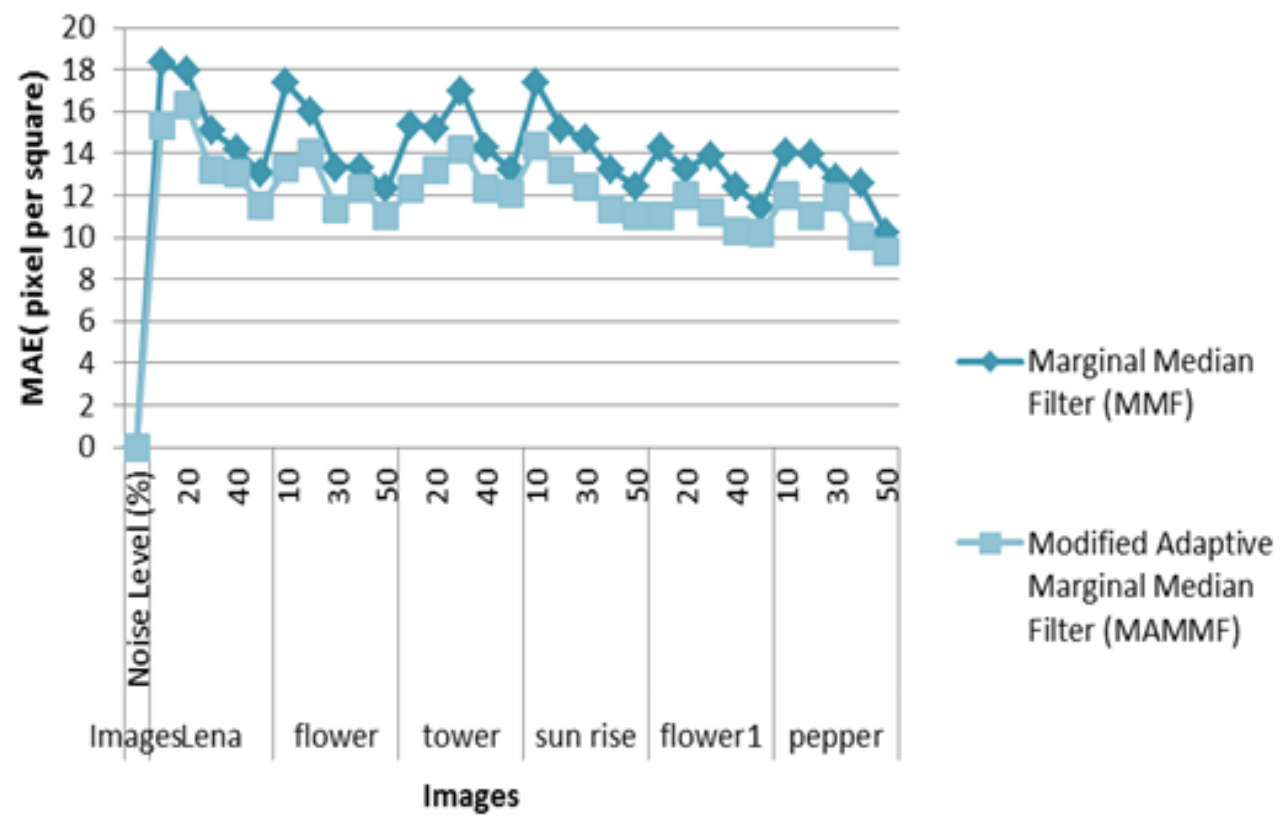

Fig. 9. MAE for different level of noise. 
From the above Table 3 shows that the combine filter provides the highest quality color image with low cost, time and low complexity. Then the output clearly shows that the proposed system provides a better result, even when we use various types of images. From the result the proposed MAMMF algorithm provides best average results such as PSNR is $43.978 \mathrm{~dB}$, MSE value is 253.73 pixel square and the MAE value is 12.2275. The PSNR, MSE and MAE values are better than the MMF such as $36.449 \mathrm{~dB}, 239.3941$ pixel square and 14.294 pixel square.

Table 3. Performance Evlaution with Combine Filter

\begin{tabular}{|c|c|l|c|c|}
\hline Parameter & \multicolumn{2}{|c|}{ Without Combine Filter } & \multicolumn{2}{c|}{ With Combine Filter } \\
\hline Scaling Method & IrfanView & $\begin{array}{l}\text { FastStone Photo } \\
\text { Resizer }\end{array}$ & IrfanView & $\begin{array}{c}\text { FastStone Photo } \\
\text { Resizer }\end{array}$ \\
\hline Input Image & $152 X 160$ & $228 X 240$ & $152 X 160$ & $228 X 240$ \\
\hline Output Image & $235 X 419$ & $312 X 456$ & $235 X 419$ & $312 X 456$ \\
\hline PSNR (db) & 34.03 & 32.361 & 37.30 & 38.056 \\
\hline MSE (pixel square) & $1.6667 \mathrm{e}+00$ & $1.2439 \mathrm{e}+00$ & $1.79 \mathrm{e}+01$ & $1.539 \mathrm{e}+00$ \\
\hline MAE(pixel square) & 13.365 & 12.036 & 10.0502 & 10.334 \\
\hline
\end{tabular}

\section{Conclusion}

Noise free images are very important in research analysis. But the images are corrupted by several processes. So that the proposed Resampling with MAMMF algorithm remove the blurring, aliasing and impulse noise from the original image. Thus the proposed algorithm produces the highest quality image by Resampling enhanced Lancozs filter with Modified Adaptive Marginal Median Filter. The PSNR and MSE values are computed from the experimental result which is used to analyze the performance of the proposed algorithm. Finally the combined filter produces the enhanced output with low cost and low memory requirements. In future the noise removal filters are combined to work with the several optimization and segmentation approach for eliminating the unwanted details in the image with efficient manner.

\section{References}

[1] Engineersgarage.

Retrieved,

from http://www.engineersgarage.com/articles/image-processing-tutorial-applications.

[2] Piotr, S. W. (2001). Fast impulsive noise removal. IEEE Transactions on Image Processing, 10(1).

[3] Shih, L. C. (2013). VLSI implementation of an adaptive edge-enhanced image scalar for real-time multimedia applications. IEEE Transactions on Circuits and Systems for Video Technology, 23(1).

[4] Anisha, K. K., \& Wilscy, M. (2011). Impulse noise removal from medical images using fuzzy genetic algorithm. An International Journal of Signal and Image Processing, 2(4).

[5] Imagemagick. Retrieved, from http://www.imagemagick.org/Usage/resize

[6] Pei, Y. C. (2010). A low-cost VLSI implementation for efficient removal of impulse noise. IEEE Transactions on Very Large Scale Integration (VLSI) Systems, 18(3).

[7] Pei, Y. C. (2010). VLSI implementation of an edge-oriented image scaling processor. IEEE Transactions 
on very Large Scale Integration (VLSI) Systems, 17(9).

[8] Chih, Y. L., Chien, C. H., \& Pei, Y. C. (2013). An efficient denoising architecture for removal of impulse noise in images. IEEE Transactions on Computers, 6(4).

[9] Shih, L. C. (2013). VLSI implementation of a low-cost high-quality image scaling processor. IEEE Transactions on Circuits and Systems, Express Brief, 60(1).

[10] Emin, M. Y., \& Erkan, B. (2004). A simple neuro-fuzzy impulse detector for efficient blur reduction of impulse noise removal operators for digital images. IEEE Transactions on Fuzzy Systems, 12(6).

[11] Jayamanmadharao, M., Ramanaidu, M. S., \& KVVS, R. (2013). Impulse noise removal from digital images - A computational hybrid approach. Global Journal of Computer Science and Technology Graphics and Vision, 13(1).

[12] Safinaz, S. (2014). An efficient algorthm for image scaling with high boost filtering. International Journal of Scientific and Research Publications, 4(5).

[13] Shashank, S., \& Tarun, G. (2014), Upscaling capsule endoscopic low resolution images. International Journal of Advanced Research in Computer Science and Software Engineering, 4(5).

[14] Manohar, K., \& Balaji, S. (2013). Literature survey on impulse noise reduction. An International Joural on Signal and Image Processing, 4(5).

[15] Samuel, M., Valent'ın, G., \& Almanzor, S. (2011). Adaptive marginal median filter for colour images.

[16] Ajay, K. N., Surbhi, S., Shailender, G., \& Bharat, B. (2014). A comparative study of mixed noise removal techniques. International Journal of Signal Processing, Image Processing and Pattern Recognition, 7(1), 405-414.

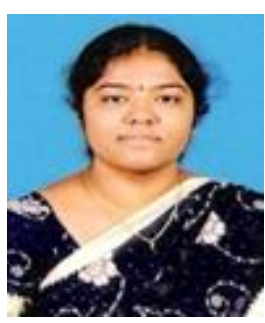

S. Arul Jothi is a research scholor at Sri Ramakrishana Engineering College, Coimbatore. She holds a master's degree in eelectronic and commnication engineering and a bachelor's degree in electronic and communication engineering. She is currently pursuing her research in image processing and VLSI.

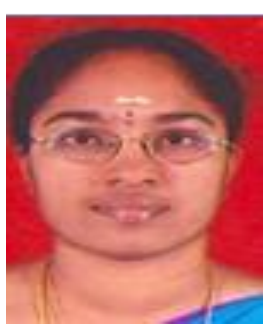

N. Santhiya Kumari is an associate proffessor at Knowlegde Institue of Technology in Salem. She holds a master's degree in eelectronic and commnication engineering and a bachelor's degree in electronic and communication. Her research area includes the image processing.

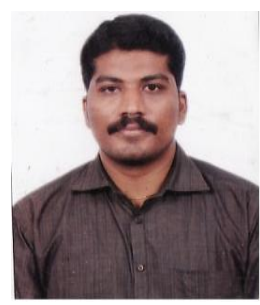

M. Ramkumar Raja is an assistant professor at Coimbatore Institue of Engineering and Technology, Coimbatore. He holds the master degree in electronic communication engineering and a bachelor's degree in electronic communication. His research interest include the image processing. pursuing his research in image processing and VLSI. 